Article

\title{
Deep Learning for COVID-19 Recognition
}

\author{
Yuchen Zhang ${ }^{1, *}$, Yanyan Zhang ${ }^{1}$ \\ 1 Department of Electronic and Information Engineering, Institute of Intelligent Network and Information \\ System, Nanjing University of Information and Science Technology, Nanjing, 210044, China; \\ 002243@nuist.edu.cn \\ * Correspondence: erik.yczhang@gmail.com
}

\begin{abstract}
Pneumonia is a leading cause of death worldwide, and one of the most significant approaches to diagnose pneumonia is Chest X-ray (CXR) since it was used in clinical scenes. Convolutional neural networks (CNNs) have been widely used in computer vision community. Along with the development of CNNs, we want to make use of CNNs to recognize CXR of people who get pneumonia and make classification. It is important, especially during epidemic period. In this paper, we present a new type of residual learning framework, PEPX-Resnet, which makes use of a type of lightweight residual, and apply this network to CXR dataset. The result shows that PEPXResnet is easier to optimize and can have better results, especially for COVID-19 cases. PEPX-Resnet could reach higher accuracy, f1 score and some other evaluations for CXR dataset.
\end{abstract}

Keywords: pneumonia; Resnet; residual; PEPX-Resnet; COVID-19

\section{Introduction}

Rapid progresses have been evidenced in computer vision via deep learning and large-scale annotated image datasets [1-5], and recent researches show that the depth of network is of great importance, because deep networks could lead to good results in challenging recognition tasks. However, researchers found that deeper neural networks could cause some problems [6], and they proposed residual learning framework which is called Resnet to solve these problems, and get good results.

One of the most typical application of convolutional neural networks in computer vision is face recognition. The most popular system to recognize face are Deep Face recognize system which is shown in [4]. We have some available datasets to train and test networks, such as LFW dataset and MS-Celeb-1M [7]. These datasets have large amounts of pictures of people's faces and some related information such as their names, ages and so on.

Since scientists discovered $\mathrm{x}$-ray, the application in clinical medicine have advanced to a considerable extent. Medical imaging has developed over 50 years including Magnetic Resonance Imaging (MRI), functional magnetic resonance imaging (fMRI), Positron Emission Computed Tomography (PET), Electroencephalogram (EEG) and so on. Convolutional neural networks also be applied in this fields in [8], which is another important application of CNNs.

Pneumonia accounts for around $16 \%$ of children who died under 5 years old [9], and it has become the leading cause of death among children [10]. Coronavirus disease 2019 (COVID-19) was threatening large amounts of lives in 2020 [11-13] and COVID-19 has become a global crisis which has confirmed cases in almost any countries.

The approaches to increase the accuracy and efficiency of diagnostic has been exploited for decades [14-16]. They tried many directions, including classification [17-19], segmentation [20] and detection.

In this paper, we present a new type of residual learning framework, PEPX-Resnet, which makes use of PEPX, a lightweight residual, and this network could achieve higher accuracy, precious, sensitive and f1 score and more stable results when using it to 
recognize CXR. We will present the architecture of the network and then we will show the results of our experiments, in which we make use of PEPX-Resnet to recognize CXR and diagnose patients of COVID-19. It might be useful in clinical senses, especially when we face the outbreaks of epidemic virus which could cause pneumonia.

\section{Related work}

Image recognition have great results in recent years, and some different structures of networks have been used in some face recognition tasks. Researchers presents many approaches to deal with recognition tasks. For example, researches presented an approach to learn a projection that is at the same time distinctive and compact, achieving dimensionality reduction at the same time based on a triplet loss, and directly learns a mapping from face images to a compact Euclidean space where distances directly correspond to a measure of face similarity in [21]. [22] proposes a hybrid convolutional network (ConvNet)-Restricted Boltzmann Machine (RBM) model for face verification in wild conditions and researches showed that face recognition tasks can be well solved with deep learning and using both face identification and verification signals as supervision [23].

Along with the development of CNNs, researchers found that deeper CNNs will cause a type of degradation, which will cause decline of the results. Some researchers proposed a new architecture, Resnet, which use residual to train the network and compose a type of residual learning framework, ending up that results become much better and some problems shown in ordinal CNNs, such as degradation, were solved well. This network is used in face recognize tasks in [21], which could get more extraordinary results when using it to recognize pictures in LFW and other datasets compared with VGG [24].

Some researches use deep neural networks in other fields to solve some problems. For example, some researchers use neural networks to solve clinical problems [25-27], which make the process of diagnosis become easier for doctors and save them lots of precious time, especially during epidemic period. These researchers have good ideas and get good results in their datasets. There also are some researchers use the model in $[28,29]$ to detect pneumonia, classify pneumonia and get good results. Even some researchers have made use of CNNs to diagnose COVID-19, such as researchers of COVID-Net [30]. They proposed a new architecture of networks, especially a new type of lightweight residual called PEPX, which makes a type of combination of projections and extensions. Their experiments show that this COVID-Net could get great results, especially for COVID-19 cases.

\section{Materials and Methods}

In this section, we will show our approach to solve the clinical problem, recognition CXR, to be concise, we proposed a new type of residual learning network, PEPX-Resnet, to recognize CXR and help doctors to make classification and diagnosis. We first introduce PEPX-Resnet as a whole, and compare it with Resnet. Then, we will introduce PEPX-Resnet in detail, including its blocks and PEPX. Finally, we will talk about the focal loss which will be used in our classification problem.

\subsection{PEPX-Resnet}

PEPX-Resnet is combined by many blocks shown in Figure 5. The whole architecture is shown in Figure 1. Clearly, the network has four main blocks, and we use 3x3 kernel in these blocks. This network is mainly inspired by Resnet (Figure 3). We tried using residual in a different way, and tried different approaches to combine residuals and the backbone of the networks. 


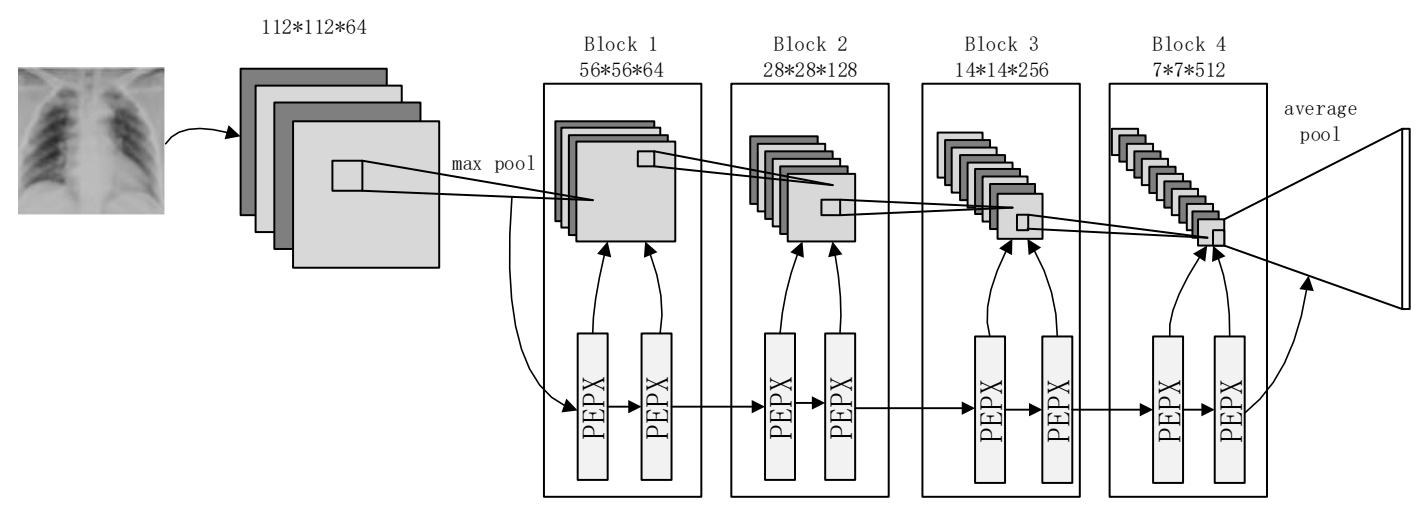

Figure 1. The architecture of PEPX-Resnet is a figure.

It is clear in Figure 1 that PEPX was not only added in each block, but also could be interpreted as a network, which means that the whole network could be interpreted as the combination of two networks, and the results of these two networks was added together with different weights. Plus, it could be observed in Figure 1 that the combination of blocks could give the whole network much more different potential architectures, because different weights of residuals and PEPX mean different architectures, even some architectures totally unsimilar.

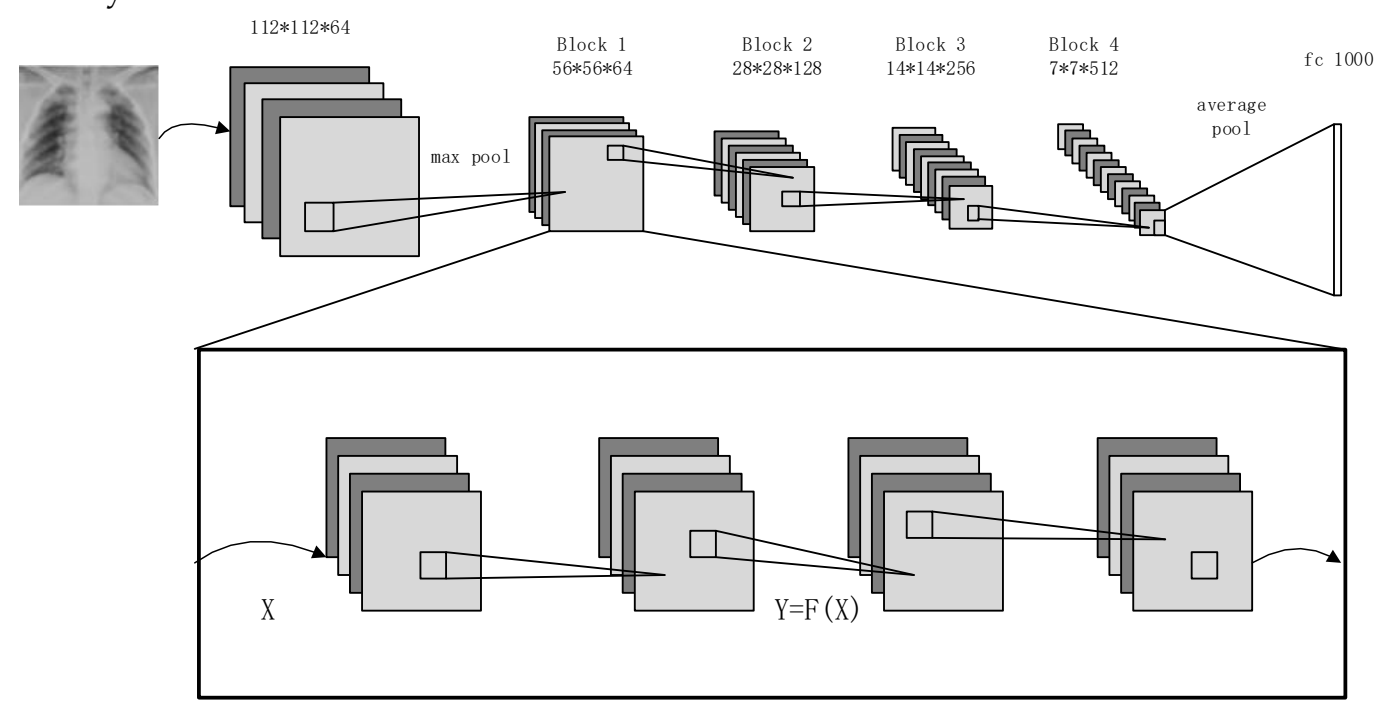

Figure 2. The architecture of a flat neural network. 


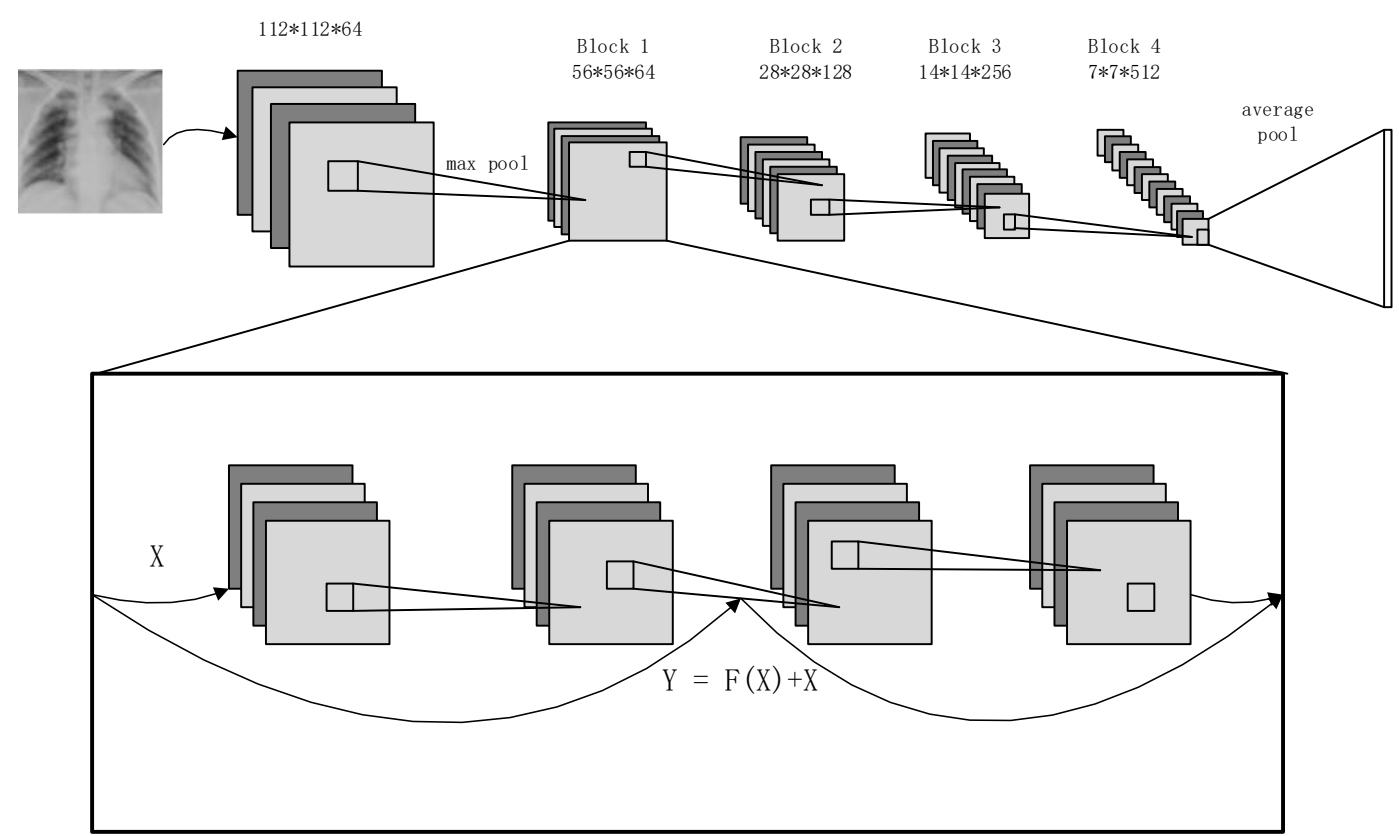

Figure 3. The architecture of Resnet.

In Figure 2 and Figure 3, we can see that Resnet adds a residual in each unit, and the backbone of the network does not change. This bottleneck residual structure improves the performance a lot, but it cannot get good results in CXR datasets. Compared with Fig 1, it is clear that the backbone of PEPX-Resnet is changed by a type of special operation, PEPX, which makes up an individual network, and the whole network allows one-direction communication of data between the backbone and the PEPX, to be concise, data just could be transferred from PEPX to convolution layers. Plus, in Figure 1 and Figure 3, we can observe that PEPX-Resnet also has large-scale residuals, which connect more convolution layers rather than Resnet.

\subsection{Blocks of PEPX-Resnet}

Let's consider $\mathrm{Y}$ is the result of a block in CNNs, the result could be written as $\mathrm{Y}=$ $F(x)$. In Resnet, researchers present a new architecture and make use of residual to make the results of the network become batter. In this structure, we consider the result of a unit in a block is $\mathrm{Y}$, and then add the result of the previous unit, then, the result of this unit could be written as $\mathrm{Y}=F(x)+x$. The added $x$ is the residual of the unit. The structure of this unit is shown in Figure 4.

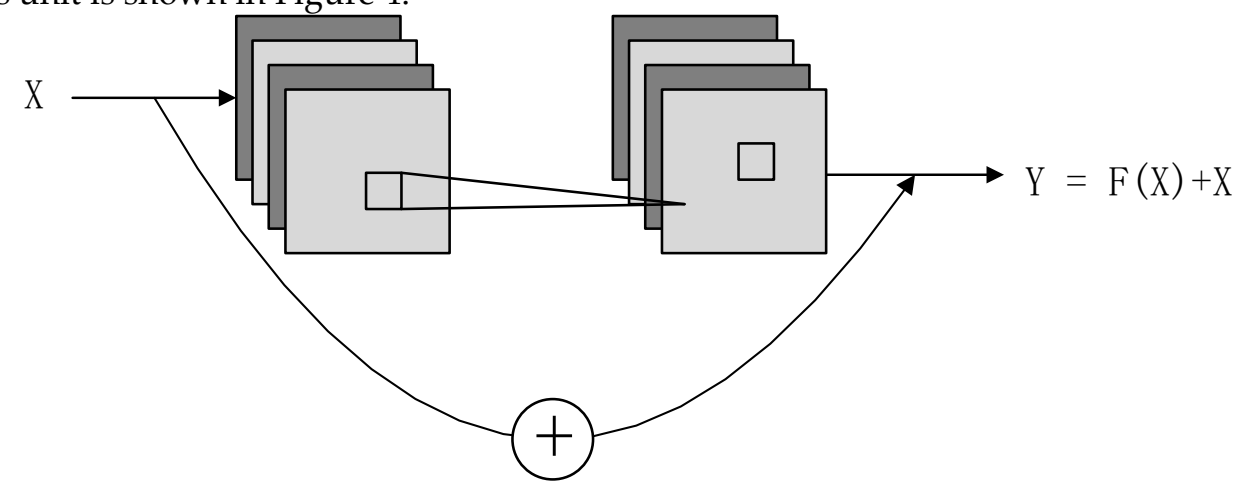

Figure 4. A unit of a block of Resnet

We modify the residual learning framework by using two level of residual and making use of a type of lightweight residual, PEPX, then we give them different weights when we add these residuals to the backbone of the network. The different weights could be 
achieved by the process of training. Let's consider the result is also $\mathrm{Y}$, and the process could be written as:

$$
\mathrm{Y}=F\left[F_{1}(x)+x\right]+A * F_{1}(x)+B * x+C * P E P X(X)
$$

$A, B$ and $C$ in the formula is the different weights of residuals and these are achieved by the process of training. These different weights could make the network have large amounts of different architectures. The basic structure of the block is shown in Figure 5:

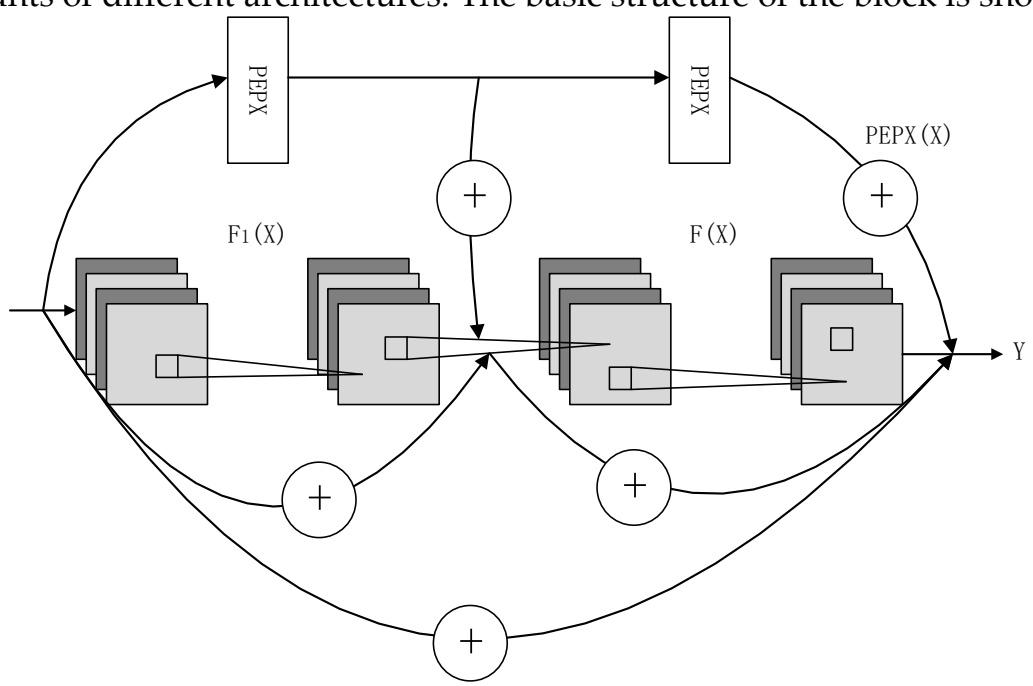

Figure 5. Building block: a building block of PEPX-Resnet

In Figure 5, the first two layers is called $F_{1}(x)$, and the last two layers is called $F(x)$. The operation in the special architecture, PEPX, is called PEPX $(X)$. We combine these operations together by adding them and give them different weights, which is gotten by the process of training. Clearly, this block has many possible architectures because of different weights. Plus, the combination of this block which was shown in Figure 1 could give the network much more different architectures.

\subsection{PEPX}

PEPX is a type of light weight residual, which was used in COVID-Net [30]. It was composed by 5 steps:

1. Project to a dimension with less channels;

2. Extend to a dimension with more channels, and the number of channels is different from input;

3. Use $3 \times 3$ kernel to detect some features;

4. Project to a dimension with the same number of channels of step 1;

5. Extend to a dimension with more channels, and the number of channels is the same as we need.

The steps of PEPX are shown in Figure 6. In this paper, we do not use depth-wise convolution to get features in step 3 as COVID-Net, because depth-wise convolution uses individual kernels in different channels, which means that depth-wise convolution cannot get special features at a location in different channels.

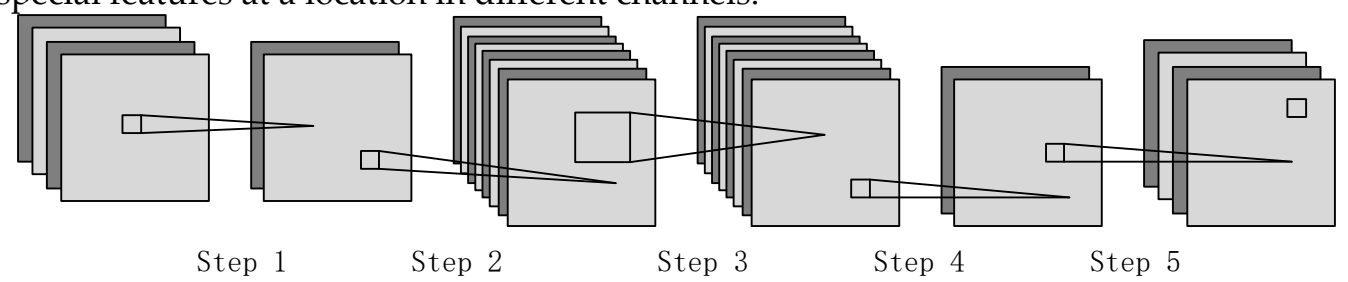

Figure 6. Steps in PEPX

\subsection{Focal loss}


Focal loss was proposed to emphasize the training of samples that cannot be predicated well, and makes the results better. The focal loss function is shown there:

$$
\mathrm{LS}=-\alpha\left(1-P_{t}\right)^{\gamma} \log P_{t}
$$

In this formula, $P_{t}$ is the probability of being classified correctly, which is gotten by softmax. This loss function could emphasize training of samples which have lower $P_{t}$, because of the coefficient $\alpha\left(1-P_{t}\right)^{\gamma}$, which will be higher when the $P_{t}$ is lower, and will be lower when $P_{t}$ is higher. By this means, when the $P_{t}$ is lower, it could make $L S$ be higher and enforce the training of these samples. The coefficient $\alpha$ and $\gamma$ have different functions. The coefficient $\alpha$ was used to solve the problem of unbalance of positive and negative data in training dataset, and coefficient $\gamma$ was used to adjust the enforcement of those samples which are classified incorrectly. In our experiments, we make $\alpha=1$, and $\gamma=1$.

\section{Experimental results}

We evaluate our network on the dataset that we get from www.kaggle.com. To be concise, from two accounts:

- $\quad$ https://www.kaggle.com/praveengovi/coronahack-chest-xraydataset;

- https://www.kaggle.com/manan2m/covidx-v3.

We could get great amounts of pictures of chest X-ray on these accounts, and these pictures included normal people and patients who get pneumonia, and especially, we also get some CXRs of patients of COVID-19. We use Resnet and PEPX-Resnet to classify these pictures into three classes:

- Normal;

- Non-COVID;

- COVID-19.

In this section, we introduce our dataset and then we compare the results of different networks.

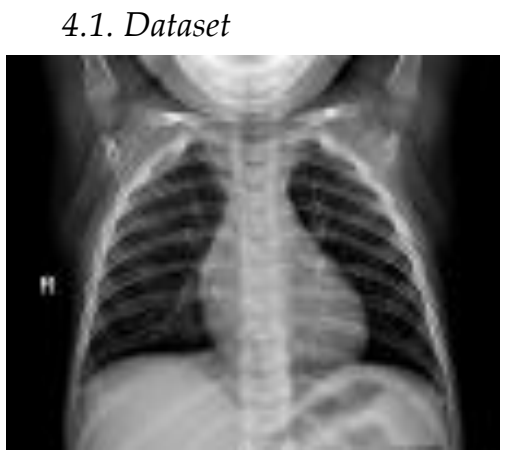

(a)

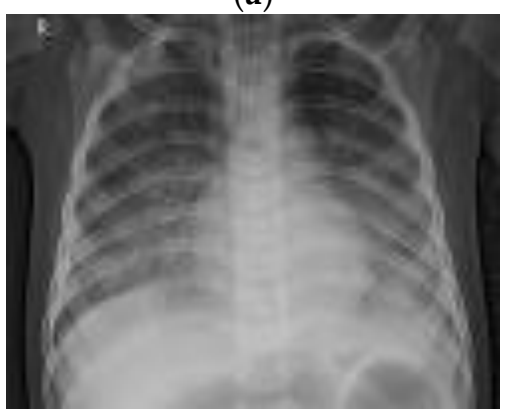

(c)

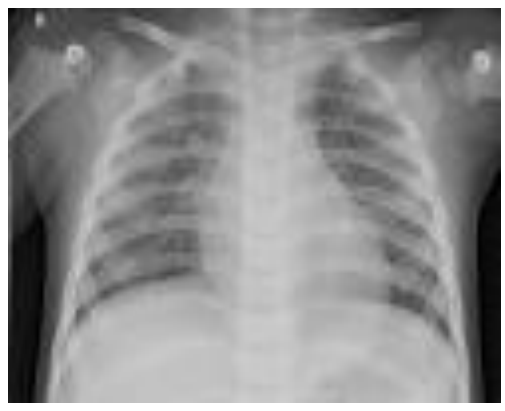

(b)

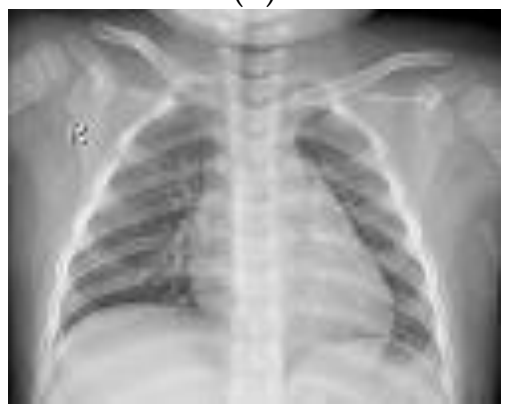

(d)

Figure 7. Some examples of CXR in dataset: (a) CXR of Normal people. (b) CXR of pneumonia caused by bacterial. (c) CXR of pneumonia caused by virus. (d) CXR of Normal people, but it still has increased opacity. 
We know that pneumonia usually manifests as an area of increased opacity in CXR. Because CXR of normal people do not have much area of opacity. Most pneumonia are caused by bacterial and virus, and it usually manifests as an area of increased opacity in CXR. However, diagnosis of pneumonia by CXR is complicated because there are many other conditions that interfere with the diagnosis of pneumonia, such as pulmonary edema, bleeding, volume reduction, lung cancer, etc. and each of these could cause increased opacity in CXR (such as (d) in Figure 7), which means that it is not an easy task to recognize pneumonia by CXR. Especially in epidemic period because of great volumes of cases, such as the outbreak of COVID-19. We can make use of our PEPX-Resnet to help doctors read these images and help them make right judgments.

We also found CXR of patients of COVID-19, which is threatening many people's lives $[17,18,19]$. The examples of these are shown in Figure 8:

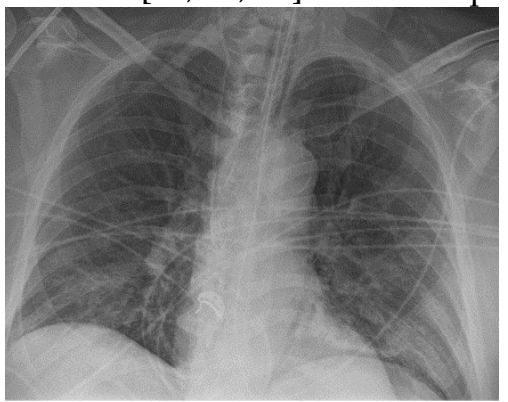

(a)

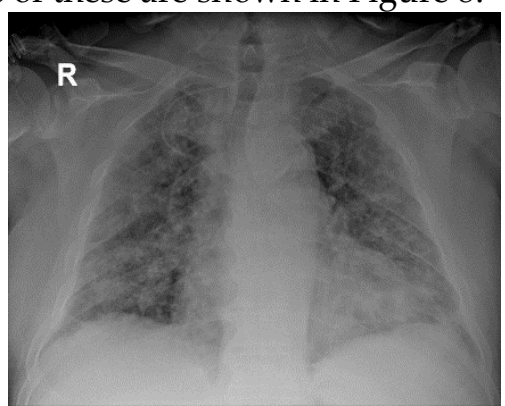

(b)

Figure 8. Some examples of CXR of COVID-19 in dataset.

In our experiments, we use 1000 CXRs of each class to train our network and use 100 CXRs of each class to test. In other words, we will use 3000 CXRs to train our networks and use 300 CXRs to test.

\subsection{Results}

We contrast the results of Resnet and PEPX-Resnet in details. We make the network make a prediction, and the prediction must be one of the results in these three classes:

- Normal;

- Non-COVID;

- COVID-19.

We also contrast the results with different loss functions including Softmax-loss and Focal loss, and the experiment shows that focal loss could get better results in our work.

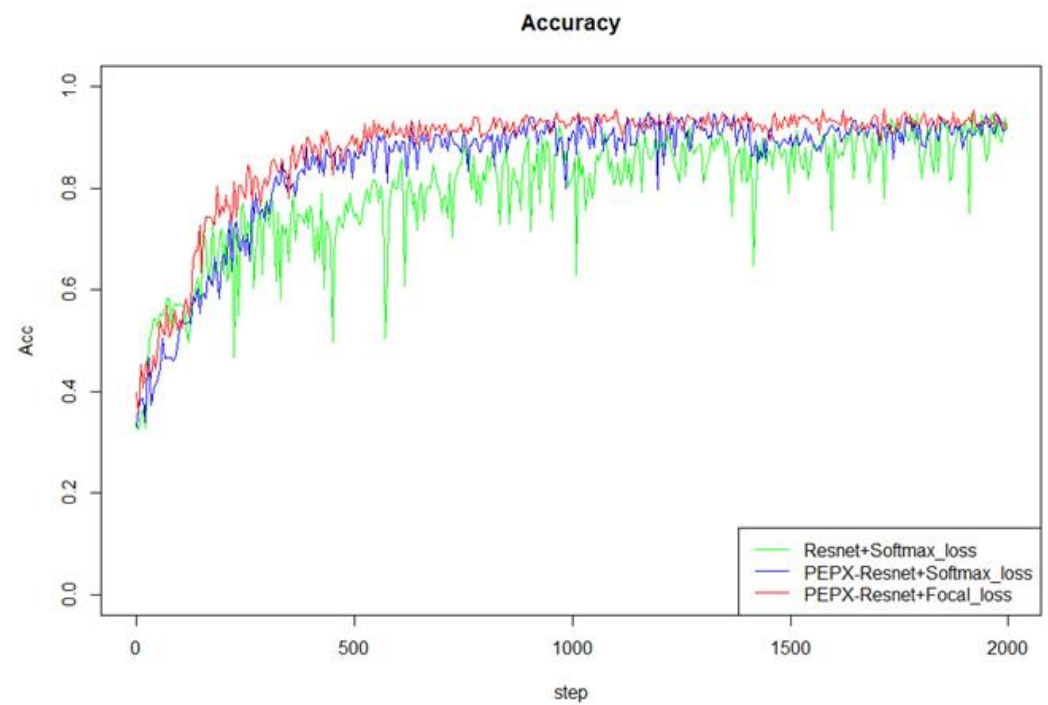


Figure 9. The accuracy of different networks and loss functions.

It is clear in Figure 9, the accuracy could be higher when we use PEPX-Resnet, especially when we make use of focal loss. By contrast, Resnet is not stable and has lots of rises and downs.

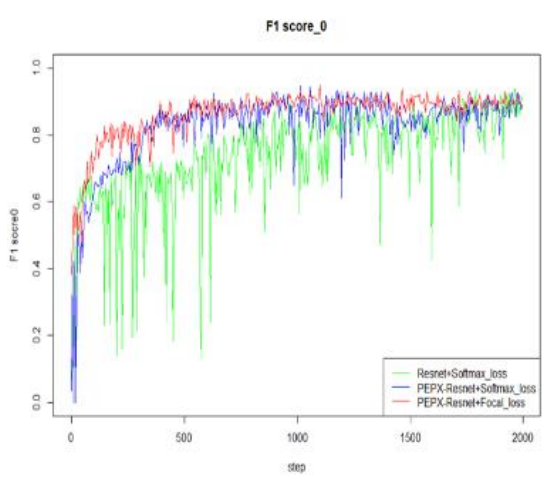

(a)

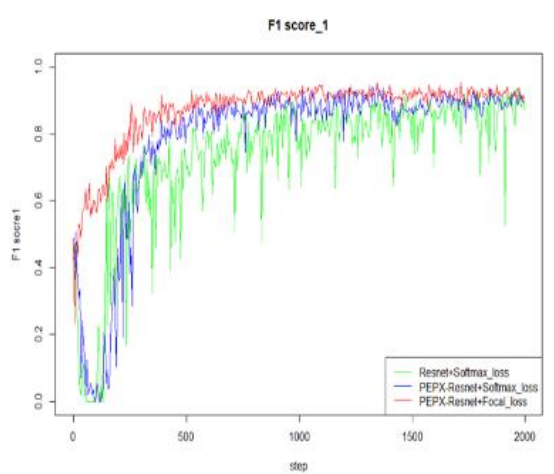

(b)

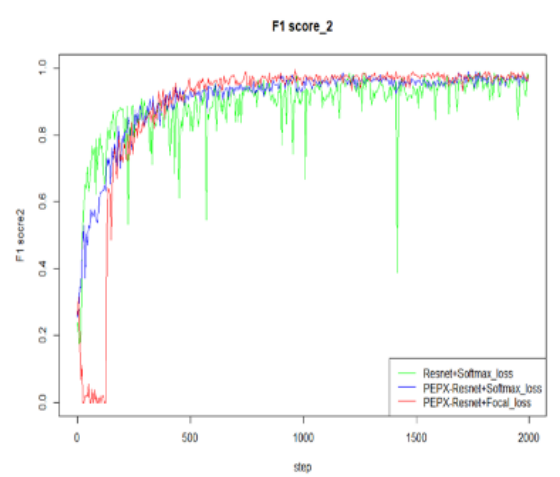

(c)

Figure 10. F1 score of different networks and loss functions.

Figure 10 shows the F1 score in our experiments, (a) in Figure 10 is F1 score of Normal, (b) in Figure 10 is F1 score of Non-COVID, (c) in Figure 10 is F1 score of class COVID19. It is easy to observe that PEPX-Resnet could have higher and more stable F1 score, especially when we make use of focal loss.

Then, we will compare different networks from many different aspects, including accuracy, precious, sensitive and F1 score. We also compare the results of PEPX-Resnet with the results of COVID-Net which was proposed in [30]. We compare the accuracy in Table 1 (The best one is bold):

Table 1. The accuracy of different networks.

\begin{tabular}{cc}
\hline & Accuracy \\
\hline Resnet18 & 0.94667 \\
COVID-Net & 0.933 \\
This work & $\mathbf{0 . 9 5 6 6 6}$ \\
\hline
\end{tabular}

Then table 2 , table 3 and table 4 will compare different networks from many different aspects, including precious, sensitive(recall) and f1 score (The best one is bold).

Table 2. Precious of different networks of these three classes.

\begin{tabular}{cccc}
\hline \multicolumn{4}{c}{ Precious $(\boldsymbol{\%})$} \\
\hline Normal & Non-COVID & COVID-19 \\
\hline Resnet18 & 0.85 & $\mathbf{0 . 9 5 2 3 7}$ & 0.96 \\
COVID-Net & 0.9047 & 0.9126 & $\mathbf{0 . 9 8 9}$ \\
This work & $\mathbf{0 . 9 6 7}$ & 0.933 & 0.97 \\
\hline
\end{tabular}

Table 3. Sensitive of different networks of these three classes.

\begin{tabular}{cccc}
\hline \multicolumn{4}{c}{ Sensitive $(\%)$} \\
\hline Normal & Non-COVID & COVID-19 \\
\hline Resnet18 & $\mathbf{0 . 9 7}$ & 0.79 & 0.97 \\
COVID-Net & 0.95 & 0.94 & 0.91 \\
\hline
\end{tabular}




\begin{tabular}{cccc}
\hline This work & 0.90 & $\mathbf{0 . 9 8}$ & $\mathbf{0 . 9 9}$ \\
\hline
\end{tabular}

Table 4. F1 score of different networks of these three classes.

\begin{tabular}{cccc}
\hline \multicolumn{3}{c}{ F1 score } \\
\hline Normal & Non-COVID & COVID-19 \\
\hline Resnet18 & 0.906 & 0.8636 & 0.9649 \\
COVID-Net & 0.9268 & 0.9260 & 0.9478 \\
This work & $\mathbf{0 . 9 3 2}$ & $\mathbf{0 . 9 5 5 9}$ & $\mathbf{0 . 9 7 9}$ \\
\hline
\end{tabular}

Clearly, PEPX-Resnet could get the best results. Even though other networks, including Resnet18 and COVID-Net could get higher precious or sensitive, when we compare F1 score, which could evaluate the network comprehensively, PEPX-Resnet gets the best results in all classes.

\section{Discussion}

In this paper, we proposed PEPX-Resnet and we use this network to recognize and classify CXRs to help doctors to handle with some clinical situation. By using two levels of residuals, a type of operation, PEPX, to calculate our data in different dimensions, we could improve accuracy, precious, sensitive and f1 score. Plus, we could make them be more stable. These make the network be more practical in clinical situation. We also tried Softmax-loss and focal loss, and the results shows that even though the accuracy did not change a lot, the loss was much more stable with focal loss, and it could help us improve some evaluations, such as F1 score. Finally, we show the results of our experiments, it is clear that PEPX-Resnet could get better results than Resnet18 and COVID-Net.

Author Contributions: Conceptualization, Yuchen Zhang; methodology, Yuchen Zhang; software, Yuchen Zhang; validation, Yuchen Zhang, and Yanyan Zhang; formal analysis, Yuchen Zhang; investigation, Yuchen Zhang; resources, Yuchen Zhang; data curation, Yuchen Zhang; writing-original draft preparation, Yuchen Zhang; writing-review and editing, Yanyan Zhang; visualization, Yuchen Zhang; supervision, Yanyan Zhang; project administration, Yanyan Zhang; funding acquisition, Yanyan Zhang. All authors have read and agreed to the published version of the manuscript.

Funding: This research was funded by National Nature Science Foundation of China, grant number 61705109 and National Nature Science Foundation of China, grant number 61931004

Acknowledgments: The research work was supported by: National Nature Science Foundation of China (No: 61705109); National Nature Science Foundation of China (No: 61931004); A Project Funded by the Priority Academic Program Development of Jiangsu Higher Education Institutions. Jiangsu Province College Students Practice and Jiangsu Innovation \& Entrepreneurship Group Talents Plan.

Conflicts of Interest: The authors declare no conflict of interest.

\section{References}

1. Y. LeCun; B. Boser; J. S. Denker; D. Henderson; R. E. Howard; W. Hubbard; and L. D. Jackel. Backpropagation applied to handwritten zip code recognition.Neural computation, 1989.

2. Parkhi O.M.; Vedaldi A; Zisserman A. Deep face recognition. Proceedings of the British Machine Vision 2015, vol. 1, no. 3, p. 6

3. Wang X; Peng Y; Lu L; Lu Z; Bagheri M; Summers RM. ChestX-ray8: Hospital-scale Chest X-ray Database and Benchmarks on Weakly-Supervised Classification and Localization of Common Thorax Diseases. IEEE CVPR 2017

4. M. Everingham; S. M. A. Eslami; L. J. Van Gool; C. Williams; J. Winn; and A. Zisserman. The pascal visual object classes challenge: A retrospective. International Journal of Computer Vision 2015 pages 111(1): 98-136.

5. A. Krizhevsky; I. Sutskever; and G. E. Hinton. Imagenet classification with deep convolutional neural networks. Advances in neural information processing systems 2012, pages 1097-1105.

6. Kaiming He; Xiangyu Zhang; Shaoqing Ren; Jian Sun. Deep Residual Learning for Image Recognition. IEEE CVPR 2016.

7. Yandong Guo; Lei Zhang; Yuxiao Hu; Xiaodong He; Jianfeng Gao. MS-Celeb-1M: A Dataset and Benchmark for Large-Scale Face Recognition. ECCV 2016. 
8. Tatiana Gabruseva; Dmytro Poplavskiy; Alexandr Kalinin. Deep Learning for Pneumonia Detection. IEEE/CVF Conference on Computer Vision and Patten Recognition Workshops, 350-351, 2020.

9. World health organization: World pneumonia day 2018. Available Online: www.who.int/maternal child adolescent/child/worldpneumonia-day-2018/en/, (accessed on 225 2018)

10. White paper: Top 20 pneumonia facts. Available Online: www.thoracic.org/patients/patient-resources/resources/toppneumonia- facts.pdf., (accessed 225 2019).

11. Wallet. Panton-valentine leukocidin-secreting staphylococcus aureus pneumonia complicating COVID-19. Emerging Infectious Diseases 2020 26(8).

12. Zou; Linghua Li; and Yuelong Shu. Clinical characteristics of coronavirus disease 2019 (COVID-19) in china: A systematic review and metaanalysis. Journal of Infection 2020.

13. Johns Hopkins Coronavirus Resource Center. Covid-19 dashboard by the center for systems science and engineering (csse) at johns hopkins university (jhu). Available Online: https://www.arcgis.com/apps/opsdashboard/index.html, (accessed 2611 2020).

14. Norliza Mohd; Noor; Omar Mohd; Rijal; Ashari Yunus; and S.A.R. Abu-Bakar. A discrimination method for the detection of pneumonia using chest radiograph. Computerized Medical Imaging and Graphics 2020, 34(2):160-166.

15. Coelho; and Ana L'ucia S. S. Andrade. Computer-aided diagnosis in chest radiography for detection of childhood pneumonia. International Journal of Medical Informatics 2008 77(8):555-564.

16. Pranav Rajpurkar, Jeremy Irvin, Kaylie Zhu, Brandon Yang, Hershel Mehta, Tony Duan, Daisy Ding, Aarti Bagul, Curtis Langlotz, Katie Shpanskaya, Matthew P. Lungren, and Andrew Y. Ng. Chexnet: Radiologist-level pneumonia detection on chest x-rays with deep learning. arXiv 2017, arXiv: 1711.05225v1.

17. Alexander Rakhlin; Alexey Shvets; Vladimir Iglovikov; and Alexandr A. Kalinin. Deep convolutional neural networks for breast cancer histology image analysis. arXiv 2018, arXiv:1802.00752 [cs.CV].

18. H. R. Roth; L. Lu; A. Seff; K. M. Cherry; J. Hoffman; S. Wang; J. Liu; E. Turkbey; and R. M. Summers. A new 2.5D representation for lymph node detection using random sets of deep convolutional neural network observations. arXiv 2014. arXiv:1406.2639v1 [cs.CV].

19. H. Shin; H. Roth; M. Gao; L. Lu; Z. Xu; I. Nogues; J. Yao; D. Mollura; and R. Summers. Deep convolutional neural networks for computer-aided detection: Cnn architectures, dataset characteristics and transfer learnings. IEEE Trans. Medical Imaging 2016. 35(5):1285-1298

20. Olaf Ronneberger; Philipp Fischer; and Thomas Brox. Unet: Convolutional networks for biomedical image segmentation. arXiv 2015. arXiv:1505.04597v1 [cs.CV].

21. Schroff F; Kalenichenko D; and Philbin J. Facenet: a unified embedding for face recognition and clustering. Proceedings of the IEEE Conference on Computer Vision and Pattern Recognition (CVPR), pp. 815-823 2015.

22. Sun Y; Wang X; and Tang X. Hybrid deep learning for face verification. Proceedings of the IEEE International Conference on Computer Vision (ICCV), 2013, pp. 1489-1496.

23. Sun Y; Chen Y; Wang X; and Tang X. Deep learning face representation by joint identification-verification. arXiv 2014. arXiv:1406.4773v1 [cs.CV].

24. K. Simonyan; and A. Zisserman. Very deep convolutional networksfor large-scale image recognition. arXiv 2015. arXiv:1409.1556v6 [cs.CV].

25. Yubing Li; Xuezhong Zhou; Runshun Zhang; Yinghui Wang; Jingqing Hu; Qi Xie; and Baoyan Liu. Complex network approach for analyzing TCM clinical herb-symptom relationships. 2013 IEEE International Conference on Bioinformatics and Biomedicine, 2013, pp. 116-117, doi: 10.1109/BIBM.2013.6732652.

26. H. Dickhaus. Wavelet neural networks for clinical diagnosis. 2001 Conference Proceedings of the 23rd Annual International Conference of the IEEE Engineering in Medicine and Biology Society, 2001, pp. 4095 vol.4-, doi: 10.1109/IEMBS.2001.1019754.

27. G.M. Papadourakis; E. Gaga; and G. Vareltzis. Use of artificial neural networks for clinical decision-making (Maldescensus testis). [Proceedings 1992] IJCNN International Joint Conference on Neural Networks. 1992, pp. 159-164 vol.3, doi: 10.1109/IJCNN.1992.227176.

28. Lin T.Y.; Goyal P.; Girshick R.; He K.; and Dollr P. Focal loss for dense object detection. IEEE International Conference on Computer Vision (ICCV), page 29993007, 2017.

29. J. Deng; W. Dong; R. Socher; L.-J. Li; K. Li; and L. Fei-Fei. ImageNet: A Large-Scale Hierarchical Image Database. 2009 IEEE Conference on Computer Vision and Pattern Recognition, 2009, pp. 248-255, doi: 10.1109/CVPR.2009.5206848.

30. L. Wang; A. Wong and Alexander Wong. COVID-Net: A Tailored Deep Convolutional Neural Network Design for Detection for COVID-19 Cases from Chest Radiography Images," Sci Rep, 2020. https://doi.org/10.1038/s41598-020-76550-z. 